\title{
Outcomes of Non-Metastatic Gestational Trophoblastic Neoplasia: Twelve Year Experience from a Northern Thailand Tertiary Care Center
}

\author{
Prapaporn Suprasert*, Manatsawee Manopunya
}

\begin{abstract}
Gestational trophoblastic neoplasia (GTN) is the malignant form of gestational trophoblastic disease. In non-metastatic GTN, the outcomes of treatment are impressive with methotrexate (MTX) or actinomycin D. We retrospectively reviewed the outcomes of non-metastatic GTN treated at our center from January, 1999 to December, 2013. One hundred and nine patients were recruited to the study. The median age was 33.1 years and over $90 \%$ were referral cases. Abnormal vaginal symptoms developed in $37.6 \%$ while $56.4 \%$ were asymptomatic. The most common antecedent pregnancy was a complete mole $(92.7 \%)$ with the median interval time from antecedent pregnancy to GTN development being 2.0 months. The median pretreatment B-hCG was 5,624 mIu/ $\mathrm{ml}$. The most common first line treatment was methotrexate (MTX) and folinic acid (91.7\%) followed by weekly MTX (4.6\%), etoposide+ MTX+actinomycin D (EMA) (2.8\%), and actinomycin D (0.9\%), with the median number of cycles at 5.0. The positive response to first line chemotherapy was $73.8 \%$. The patients were given subsequent chemotherapeutic regimens after resistance to the first line therapy and showed a final remission rate of $89.9 \%$. The significant factor that was frequently found in patients who were non-responders to the first line treatment was a hysterectomy procedure. Two patients developed lung metastasis and brain metastasis at one and four years after the first treatment, respectively. In conclusion, the outcomes of non-metastatic GTN were excellent. However, the patients need long term follow up due to the possibility of developing multiple organ metastases.
\end{abstract}

Keywords: Non-metastatic gestational trophoblastic neoplasia - outcomes - treatment

Asian Pac J Cancer Prev, 16 (14), 5913-5916

\section{Introduction}

Gestational trophoblastic neoplasia (GTN) is a rare malignant form of gestational trophoblastic disease. It is classified as either invasive mole, gestational choriocarcinoma, placental site trophoblastic tumor (PSTT) or epithelioid trophoblastic tumor (ETT) (Froeling and Seckl, 2014). Both invasive mole and gestational choriocarcinoma occur more frequently and respond very well to various types of chemotherapy whereas PSTT and ETT are extremely rare and usually resistant to chemotherapy (Biscaro et al., 2015). FIGO suggested that GTN should include only invasive mole and gestational choriocarcinoma due to their similar characteristics (Ngan et al., 2012). The most common antecedent pregnancy of GTN is hydatidiform mole which is found as high as $60 \%$ followed by abortion (30\%) and term pregnancy or ectopic pregnancy (10\%) (Ngan et al., 2012). The diagnosis of GTN consisted of the rising or plateau of B-hCG after a treated molar pregnancy or presentation with a high level of B-hCG in women with multiple organ metastasis especially lungs and brain even without the history of a molar pregnancy
(Ngan et al., 2012; Biscaro et al., 2015). The management of GTN was dependent on the WHO risk scoring system that defined cases as low and high risk. The low risk cases included GTN patients with the disease confined to the uterus or metastasis to gynecologic organs or lung with a WHO total risk score less than seven. High risk was defined as the GTN patients with WHO total risk score of equal or more than seven. Normally, in patients with a low risk, single chemotherapy with methotrexate (MTX) or actinomycin D showed very impressive outcomes while patients with a high score needed a combination of chemotherapy (Ngan et al., 2012; Froeling and Seckl, 2014; Biscaro et al., 2015). Nowadays, many publications have reported the outcomes of treatment in GTN patients with low risk WHO score that included both non-metastatic and metastatic GTN patients (Gilani et al., 2013; Ozalp et al., 2014; Taylor et al., 2015). There was, however, limited data regarding the treatment of non-metastatic GTN patients. We conducted this retrospective review to identify the outcomes for this group of patients treated over twelve years at our center. These results will be beneficial for the improvement of treatment in this rare disease. 


\section{Materials and Methods}

After approval by the local Ethics Committee, the patients who were diagnosed as non-metastatic GTN treated at Chiang Mai University Hospital between January, 1999 and December, 2013 were retrospectively reviewed. The diagnosis of GTN used standard criteria that consisted of the plateau level of B-hCG for three weeks or rising more than $10 \%$ for at least 2 weeks after a treated molar pregnancy or by histology that showed choriocarcinoma from hysterectomy or uterine curettage (Ngan et al., 2012; Seckl et al., 2013). The patients with PSTT and ETT were excluded. After being diagnosed as GTN, the patients received a complete physical and pelvic examination and further investigation with chest- X-ray and/or pelvic ultrasound. If all the results were normal except the uterine lesion, the patients were classified as non-metastatic GTN. The treatment depended on the attending physician.

The basic clinical data, the antecedent pregnancy, the histology, the level of pretreatment B-hCG, the pelvic ultrasonography results, the type and cycle of chemotherapy, the surgical intervention and the outcomes of treatment were collected. Descriptive data of all studied patients were presented as median with range and discrete data were noted as number and percentages. The Chisquare test, the Fisher Exact test and logistic regression multivariate analysis were used to evaluate the statistical significance of the prognostic factors for response to first line chemotherapy. The statistical significance was noted when a P-value was less than 0.05. SPSS for Windows program (Version 17.0, Chicago, IL, USA) were used to analyze the statistical data.

\section{Results}

One hundred and nine patients were recruited to the study. The basic clinical data was noted in Table 1 . The median age was 31.0 years old with a range from 15-54 years old. About half of the participants lived in Chiang Mai. However, over $90 \%$ of the patients were referral cases and of these patients, 8 cases received chemotherapy with methotrexate (MTX) at their primary care hospital for treatment in six patients and for prophylaxis in the other two cases.

Nearly $60 \%$ of the studied patients were asymptomatic whereas about one-third presented with abnormal vaginal bleeding. The most common antecedent pregnancy was a molar pregnancy with the median interval time from antecedent pregnancy to diagnosis of GTN of 2.0 months. The median pretreatment of B-hCG was 5,624/ml. About $60 \%$ of the studied patients showed abnormal uterine lesion by pelvic ultrasonography.

Regarding treatment, all of the studied patients received chemotherapy with the details presented in Table 2. About first line treatment, nearly $90 \%$ of them received high dose MTX with folinic acid while the rest were given weekly MTX, actinomycin D and etoposide + MTX + actinomycin D (EMA) in five, one and three patients, respectively. All three patients who received EMA were diagnosed as choriocarcinoma from the uterine curettage specimen and responded very well from this regimen. Only 103 patients were able to evaluate the response, the response rate of these first line treatments was $73.8 \%$ (76 cases).

The non-responding patients (27 cases) received second line treatment with actinomycin D in 23 cases while the rest were given EMA, 5- fluorouracil (5FU) and etoposide, etoposide + MTX + cyclophosphamide and vincristine (EM-CO) in each. Finally, 24 patients achieved remission while the remaining three patients were alive with their disease in one case and lost to follow up in two cases.

In the 13 responder patients who received a second line chemotherapy, seven of them received a first line of chemotherapy with a high dose of MTX and folinic acid and were changed to actinomycin D due to rising of liver enzymes ( 5 cases) and severe mucositis ( 1 case) while the remaining one was changed to weekly MTX for a more convenient schedule. Of these patients, only one case developed severe lung involvement after complete second line treatment for 14 months and died before receiving further chemotherapy regimen.

In the other six patients who showed remission after first line therapy received second line treatment because of the recurrence of their disease with the median progression free interval at 22 months (10-55 months). Two of them achieved remission after receiving second line treatment with EMA and actinomycin D in each case while the other four cases achieved remission after they received third line treatment. Of these four patients, the surgical intervention was performed in two cases. One underwent a craniotomy and received whole brain radiation combined with chemotherapy: ifosfamide, cisplatin, etoposide (ICE) regimen due to lung and brain metastasis at four years after finishing first line treatment and the other one underwent a hysterectomy two years after completing their first line treatment due to rising B-hCG. The pathology showed choriocarcinoma. She received further treatment with

\section{Table 1. Basic Clinical Data $(\mathrm{N}=109)$}

\begin{tabular}{lc}
\hline Characters & $\mathrm{N}(\%)$ \\
\hline Address & $50(45.9)$ \\
Chiang Mai & $16(14.7)$ \\
Chiang Rai & $11(10.1)$ \\
Lamphun & $10(9.2)$ \\
Mae Hong Son & $22(20.2)$ \\
Other & $97(89.0)$ \\
Refer Cases & \\
Symptom & $61(56.0)$ \\
None & $41(37.6)$ \\
Vaginal Bleeding & $3(2.8)$ \\
Abdominal Pain & $4(3.7)$ \\
Other & \\
Antenatal Pregnancy & $101(92.7 \%)$ \\
Complete Mole & $4(3.7 \%)$ \\
Term Pregnancy & $3(2.8 \%)$ \\
Abortion & $1(0.9 \%)$ \\
Unknown & $2.0(1-372)$ \\
Median Interval (Range, Months) & $5,624.0(18-316,350)$ \\
Median Pretreatment B-hCG & \\
(mIU/ml) & $68(62.4 \%)$ \\
Abnormal Pelvic Ultrasonography & \\
\hline
\end{tabular}


Table 2. Chemotherapy

\begin{tabular}{|c|c|}
\hline & $\mathrm{N}(\%)$ \\
\hline \multicolumn{2}{|l|}{ First Line $(\mathrm{N}=109)$} \\
\hline $\mathrm{MTX}+\mathrm{FA}$ & $100(91.7)$ \\
\hline Atinomycin D & $1(0.9)$ \\
\hline EMA & $3(2.8)$ \\
\hline Weekly MTX & $5(4.6)$ \\
\hline Median Number of Cycles (Range) & $5.0(1-24)$ \\
\hline \multicolumn{2}{|l|}{ Second line $(\mathrm{N}=39)$} \\
\hline Actinomycin D & $30(27.5)$ \\
\hline EMA & $3(2.8)$ \\
\hline $\mathrm{MTX}+\mathrm{FA}$ & $2(1.8)$ \\
\hline 5 FlUorouracil & $1(0.9)$ \\
\hline Etoposide & $1(0.9)$ \\
\hline EM-CO & $1(0.9)$ \\
\hline Weekly MTX & $1(0.9)$ \\
\hline \multicolumn{2}{|l|}{ Third Line $(\mathrm{N}=12)$} \\
\hline Actinomycin D & $3(2.8)$ \\
\hline EMA & $4(3.7)$ \\
\hline EMA-CO & $1(0.9)$ \\
\hline PI & $1(0.9)$ \\
\hline $\mathrm{ICE}^{*}$ & $1(0.9)$ \\
\hline $\mathrm{MTX}+\mathrm{FA}$ & $1(0.9)$ \\
\hline Etoposide & $1(0.9)$ \\
\hline \multicolumn{2}{|l|}{ Fourth Line $(\mathrm{N}=5)$} \\
\hline PI & $2(1.8)$ \\
\hline EMA-CO & $1(0.9)$ \\
\hline EMA & $1(0.9)$ \\
\hline Weekly MTX & $1(0.9)$ \\
\hline \multicolumn{2}{|l|}{ Fifth Line $(\mathrm{N}=4)$} \\
\hline Actinomycin D+5 Fluorouracil & $1(0.9)$ \\
\hline EMA & $1(0.9)$ \\
\hline PI & $1(0.9)$ \\
\hline EMACO & $1(0.9)$ \\
\hline \multicolumn{2}{|l|}{ Sixth Line $(\mathrm{N}=2)$} \\
\hline Carboplatin + Paclitaxel & $1(0.9)$ \\
\hline Paclitaxel & $1(0.9)$ \\
\hline
\end{tabular}

*MTX=methotrexate, $\mathrm{FA}=$ folinic acid, $\mathrm{E}=$ etoposide, $\mathrm{M}=$ methotrexate $\mathrm{A}=$ actinomycin $\mathrm{D}, \mathrm{C}=$ cyclophosphamide, $\mathrm{O}=$ vincristine $, \mathrm{P}, \mathrm{C}^{*}=$ cisplatin, I=ifosfamide
EMA and EMA-CO regimen and achieved remission. The remaining two cases were given actinomycin $\mathrm{D}$ as third line chemotherapy and achieved remission. However, both of them developed brain infarction without definite diagnosis of GTN after completion of chemotherapy for six and seven years, respectively.

Concerning surgical intervention with hysterectomy that was done in 11 patients, we found that this procedure was performed more frequently in non-responder patients while another factor such as advanced aged, history of molar pregnancy, abnormal symptom, abnormal finding from pelvic ultrasonography and pretreatment B-hCG more than $100,000 \mathrm{mIU} / \mathrm{ml}$ were not significantly different between responder and non-responder patients as shown in Table 3. Of those patients who received a hysterectomy, eight of them were operated on after failure of first line chemotherapy. The second line and third line treatment were given in six and two patients, respectively.

Finally, with the median follow up time at 30.0 (1169) months, the remission rate of the studied patients was $89.9 \%$. There were three patients alive with disease, one patient died from lung metastasis and seven patients were lost to follow up.

\section{Discussion}

The median age, the pretreatment of serum B-hCG, the interval time before diagnosis and the antecedent pregnancy in the present study were similar to previous reports of low risk GTN (Gilani et al., 2013; Maestá et al., 2013). However, the presenting symptoms were rarely mentioned in those reports. We found that about $45 \%$ of our patients did not have any symptoms while only $30 \%$ developed abnormal vaginal bleeding even through over $60 \%$ of the patients showed abnormal uterine lesions from ultrasonographic findings. This might be from the early detection of GTN from the regular follow up schedule after treatment of molar pregnancy.

The routine metastatic work up in the present study utilized only a chest X-ray and if normal, no further CT-

Table 3. Univariate and Multivariate Analysis of Factor Associated with First Line Chemotherapy Response

\begin{tabular}{|c|c|c|c|c|}
\hline \multirow[t]{2}{*}{ Factor } & \multirow{2}{*}{$\begin{array}{c}\text { Response } \\
\mathrm{N}=76\end{array}$} & \multirow{2}{*}{$\begin{array}{l}\text { Non-response } \\
\qquad \begin{array}{c}\mathrm{N}=27\end{array}\end{array}$} & \multicolumn{2}{|c|}{$\mathrm{P}$ value } \\
\hline & & & Univariate Analysis & Multivariate Analysis \\
\hline Age $<40$ Years & $54(71.4 \%)$ & $18(66.7 \%)$ & 0.67 & 0.76 \\
\hline Age $>40$ Years & $22(28.9 \%)$ & $9(33.3 \%)$ & & \\
\hline \multicolumn{5}{|c|}{ Antecedent Pregnancy } \\
\hline Mole & $67(89.3 \%)$ & $26(96.3 \%)$ & 0.44 & 0.32 \\
\hline Other & $8(10.7 \%)$ & $1(3.7 \%)$ & & \\
\hline \multicolumn{5}{|l|}{ Symptom } \\
\hline None & $43(56.6 \%)$ & $18(72.0 \%)$ & 0.17 & 0.1 \\
\hline Present & $33(43.4 \%)$ & $7(28.0 \%)$ & & \\
\hline \multicolumn{5}{|l|}{ Pelvic Ultrasound** } \\
\hline Normal & $21(29.6 \%)$ & $9(37.5 \%)$ & 0.47 & 0.42 \\
\hline Abnormal & $50(70.4 \%)$ & $15(62.5 \%)$ & & \\
\hline \multicolumn{5}{|c|}{ Pretreatment B-hCG** } \\
\hline$<100,000 \mathrm{mIU} / \mathrm{ml}$ & $71(93.4 \%)$ & $25(96.2 \%)$ & 1 & 0.64 \\
\hline$>100,000 \mathrm{mIU} / \mathrm{ml}$ & $5(6.6 \%)$ & $1(3.8 \%)$ & & \\
\hline \multicolumn{5}{|l|}{ Hysterectomy** } \\
\hline Not Performed & $73(96.1 \%)$ & $19(70.4 \%)$ & $<0.001$ & $<0.001$ \\
\hline Performed & $3(3.9 \%)$ & $8(29.6 \%)$ & & \\
\hline
\end{tabular}

***xclude missing data 
chest was done. This strategy was like the recommendation from the European and Japanese Society of Medical Oncology (Seckl et al., 2013). In contrast to the suggestion from the New England Trophoblastic Disease Center that recommended further investigation with a CT-chest in patients who showed negative chest X-ray to detect $40 \%$ of micrometastasis that could not be identified by chest $\mathrm{X}$-ray. However, this guideline is not appropriate for our institute due to the long waiting time for CT-scan and might not be cost-effective. Gamer et al suggested from their study that micrometastasis identified only from CTchest did not alter the treatment outcomes in comparison to those patients with non-micrometastasis of lung when using a similar chemotherapy regimen (Gamer et al., 2004).

Regarding chemotherapy in non-metastatic GTN, high dose MTX and folinic acid were more frequently used as first line rather than actinomycin D due to the minimal side effects. This regimen showed little nausea and vomiting and no alopecia (May et al., 2011). However, a significant side effect of MTX was liver dysfunction and mucositis, six patients of our study had their chemotherapy changed from MTX and folinic acid to actinomycin D from these adverse events. Another form of MTX usage in GTN is weekly MTX that was more convenient for patients due to being received once a week. Gillani et al presented an impressive outcome with the remission rate over $80 \%$ of weekly MTX in low risk GTN patients. They also suggested that patients with a WHO prognostic score less than 2 were more likely to receive better outcome from this regimen (Gilani et al., 2013). In addition, some centers recommended combination chemotherapy for patients with tissue proven choriocarcinoma (Taylor et al., 2015). In the present study, three patients whose tissue diagnosis showed choriocarcinoma were given EMA with an excellent outcome.

The response rate of the first line treatment in our study was $73.8 \%$ that was in a range of previous reports that showed the remission rate as $50-90 \%$. The wide variability of response rate might be from the different in dose, interval and route of chemotherapy administration in each of the studies (Seckl et al., 2013). Chapman-Davis et al found that the factors associated with resistant to MTX in non-metastatic GTN were the choriocarcinoma histology and the more than $100,000 \mathrm{mIU} / \mathrm{ml}$ of pretreatment B-hCG (Chapman-Davis et al., 2012). However, in the present report, we did not find this to be a significant factor. Surgical intervention with hysterectomy was the only independent factor that was associated with patients who were resistant to first line chemotherapy. This might be due to the limited number of our patients to demonstrate significant factors.

The various chemotherapy regimens are reported as further treatment after failure of the first line regimen. MTX was frequently changed to actinomycin D after resistance with high success outcomes. In the present study, over $90 \%$ (24 of 27) of the non-responder patients showed remission after having received the second line treatment. However, three patients still developed multidrug resistance. Various regimens were given in these types of patients in the literature such as EMA,
EMA-CO,EMA-CE, Actinomycin D+5FU, ICE, taxol with modest outcomes (Manopunya and Suprasert, 2012; Seckl et al., 2013).

The limitation of this study was the inability to identify the FIGO prognostic score due to the missing data of the score in most cases. However, the strength of this report was the study that revealed the outcomes of non-metastatic GTN with a greater number of these patients uncommonly mentioned in the literature.

In conclusion, the outcome of non-metastatic GTN was very excellent with the first and second line treatment of chemotherapy. Hysterectomy was more frequently done in the first line chemo-resistant patients. In addition, even low risk, long term follow up was necessary due to the potential recurrence with multiple organ involvement.

\section{Acknowledgements}

We wish to thank the National Research University Project under Thailand's Office of Higher Education Commission and Chiang Mai University for the financial support in this project.

\section{References}

Biscaro A, Braga A, Berkowitz RS (2015). Diagnosis, classification and treatment of gestational trophoblastic neoplasia. Rev Bras Ginecol Obstet, 37, 42-51.

Chapman-Davis E, Hoekstra AV, Rademaker AW,et la (2012). Treatment of nonmetastatic and metastatic low-risk gestational trophoblastic neoplasia: factors associated with resistance to single-agent methotrexate chemotherapy. Gynecol Oncol, 125, 572-5.

Froeling FE, Seckl MJ (2014). Gestational trophoblastic tumours: an update for 2014. Curr Oncol Rep, 16, 408-18.

Gilani MM, Fariba B, Behtash N, et al (2013). The WHO score predicts treatment outcome in low risk gestational trophoblastic neoplasia patients treated with weekly intramuscular methotrexate. J Cancer Res Ther, 9, 38-43.

Gamer EI, Garrett A, Goldstein DP, et al (2004). Significance of chest computed tomography findings in the evaluation and treatment of persistent gestational trophoblastic neoplasia. J Reprod Med, 49, 411-4.

Maestá I, Growdon WB, Goldstein DP,et al (2013). Prognostic factors associated with time to hCG remission in patients with low-risk postmolar gestational trophoblastic neoplasia. Gynecol Oncol, 130, 312-6.

Manopunya M, Suprasert P (2012). Resistant gestational trophoblastic neoplasia patients treated with 5-fluorouracil plus actinomycin D. Asian Pac J Cancer Prev, 13, 387-90.

May T, Goldstein DP, Berkowitz RS,et al (2011). Current chemotherapeutic management of patients with gestational trophoblastic neoplasia. Chemother Res Pract, 2011, 1-12.

Ngan HY, Kohorn EI, Cole LA,et al (2012). Trophoblastic disease. Int J Gynaecol Obstet, 119, 130-6.

Ozalp SS, Telli E, Oge T, et al (2014). Multicenter analysis of gestational trophoblastic neoplasia in Turkey. Asian Pac J Cancer Prev, 15, 3625-8.

Seckl MJ, Sebire NJ, Fisher RA, et al (2013). Gestational trophoblastic disease: ESMO Clinical Practice Guidelines for diagnosis, treatment and follow-up. Ann Oncol, 24, 39-50.

Taylor F, Short D, Winter MC, et al (2015). A retrospective study to evaluate single agent methotrexate treatment in low risk gestational choriocarcinoma in the United Kingdom. Gynecol Oncol, 136, 258-63. 